\title{
IN THE CLASSROOM
}

Learn to embrace the enabling power of tech

\section{Katherine Haxton}

The role of technology in education is continually changing. As technology enables us to record whole lecture courses with ease, shift from pen and paper to electronic note taking and engage learners in new and innovative ways, how do we embrace technology without fearing it?

We do not fully understand how people learn. We have theories, ideas, techniques and methodologies that seem to work in specific contexts and circumstances. Our teaching strategies evolve and adapt to our changing understanding of how people acquire knowledge, drawing ideas from diverse sources and occasionally undergoing a significant jolt from a transformative idea or technology. There are a few things we can all agree on: there is no one-size-fits-all method for learning that we know of; technology is not going away any time soon; and, human nature being what it is, we need to be strategic in our teaching and learning for the greatest benefit.

Once upon a time, the printing press was a transformative technology. Gone were the days of meticulously transcribing manuscripts by hand, or the recording and transmission of knowledge in songs or fireside stories. What a difference to the human condition textbooks made: a portable source of information that anyone with a specific skill could access.

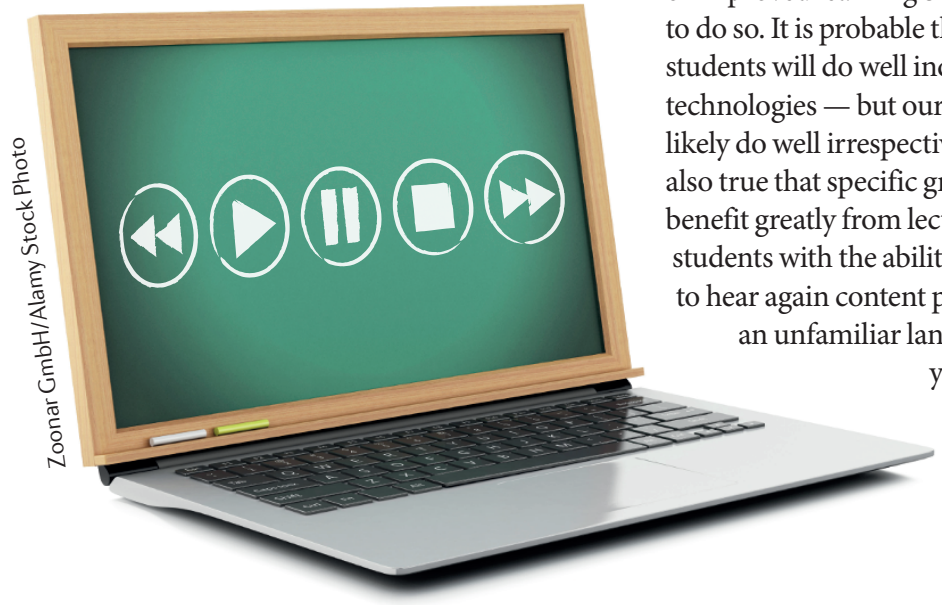

Did scholars of the time lament this technology in the manner we turn on lecture capture, PowerPoint or the presence of laptops in the classroom?

Recording lectures is not a new thing. Audio recordings, video recordings and multimedia recordings have been made and distributed for decades. How else would we have access to Richard Feynman's lectures from the last century? Lecture capture technologies have evolved and now it is commonplace for lectures to be recorded and distributed to students. They get the benefits of the live performance and the box-set recording. We do not fully understand the impact that this has on how our students learn but that is true of everything we do. We do know that it is very popular with students - an academic safety blanket if you will. We also know that the technology required represents a substantial investment for any institution, with options ranging from screen capture to full performance recording. We may question such an investment in the absence of concrete evidence of improved learning but we would be wrong to do so. It is probable that our most able students will do well independently of such technologies - but our most able students will likely do well irrespective of what we do. It is also true that specific groups of students may from lecture capture. Providing students with the ability to review concepts, hear again content presented to them in yes, the facility to miss sessions (while attending to health matters or caring responsibilities) may be invaluable.
Perhaps the fact that a student who does attend a lecture may be under less pressure to take comprehensive notes in a one-off setting is sufficient reason to offer a recording.

Aside from any potential impact on learning outcomes, concerns around lecture capture tend to fall into two main categories. The first is that students will stop attending the live event. It is astonishing that teaching staff are so insecure in their teaching that they would so easily dismiss the benefit and opportunities that only first-hand attendance can bring. Staff fear for their jobs, wondering if they might eventually be replaced by recordings. Although the idea of lectures being replaced by a cinematic experience may be an interesting thought experiment, were such a replacement so easy it would say more about the nature of the lecture than anything else. With our increasing knowledge of the benefits of active learning and techniques such as peer instruction, a 'traditional lecture' is rarely a justifiable use of contact time. Secondly, there is a concern that students will binge watch lectures and, as a result, learn only superficially. Such superficial learning may manifest itself in students parroting the exact wording used by the lecturer, but this is surely no different to students emulating the exact words of the lecture notes or prescribed texts. I would argue that it is a student's right to decide on the extent to which they wish to engage with learning, which may well fly in the face of conventional wisdom. In the UK, where tuition fees are a relatively recent introduction, it is often argued that through this consumerization of higher education, students will attend and study hard because they are paying for the experience and want value for money. If we follow that argument to a logical end point, the customer is always right and students can indeed decide the extent to which they engage. Students who are inclined only to engage superficially with their studies have always existed. The battle to convince them of another, better approach seems unlikely to be tilted one way or another by the existence of a lecture recording. The development of deeper approaches to learning and good study practices takes time and students may take even longer than staff to convince!

A confession here is appropriate: I can no longer take lecture notes at the speed I did as an undergraduate and would thus welcome lecture capture. My days of faithfully taking 
down 14 sides of classical mechanics derivations per hour is long past. This probably says quite a bit about why I am now a chemist. I would simply be too overwhelmed by the act of writing out an hours' worth of chalk and talk, and taking notes on the verbal explanations given, to be able to simultaneously monitor my understanding sufficiently to ask thoughtful questions or to seek clarification.

As a community, decades after their introduction, we are still debating the impact of presentation software such as PowerPoint or Keynote. Discussion of the utility of overhead projectors has tailed off along with their use. But many of these debates are eerily familiar and resonate strongly with discussions around lecture capture.

Humans are blessed with intrinsic adaptability, and the most versatile among us have a heady arsenal of tools to use in learning. Students need our support and guidance in selecting the most effective tools for their course and their individual needs, but we need to appreciate that preferences change. One of the most frustrating things about the learning styles debacle was its apparent aim of restricting students to only one or two preferred styles of learning. I have had capable students inform me that a certain mode of teaching did not suit them because they had completed a learning styles test. Learning styles was then, and remains, utter nonsense. We must, however, consider students who have genuine restrictions on their adaptability. This might be because of disability or specific educational needs, neither of which should limit their potential to achieve in chemistry, but the system of arbitrary rules and procedures in which they find themselves often does. One particularly pernicious fad is that of banning smart phones and laptops in teaching sessions. Again, I feel this says more about the insecurity
Cf Although the idea of lectures being replaced by a cinematic experience may be an interesting thought experiment, were such a replacement so easy it would say more about the nature of the lecture than anything else

of teaching staff than the actions of students. While it may be daunting to face a classroom in which many students are shielded by a laptop screen, it is equally daunting to face a lecturer hiding behind a podium. I share concerns that laptops might distract other students, but it is egotistical to believe they must be gossiping about the lecturer. If a teaching session is valuable to the students, they will pay attention. If many are using the time to do other things, then we must take a hard look at the system we have established for their learning and ask some difficult questions about why this is happening. Why are students working on an assignment for another class during a lecture? Why, sometimes, do they feel so overwhelmed with learning that they cannot face another hour of content delivery and choose instead to play games on their phone? We may just as well ask why staff take laptops and tablets to meetings and use the time to catch up on email, marking or messaging their significant others.

Be it lecture capture, presentation software or laptops, blanket bans are never appropriate and indeed harm students who rely on their enabling power. Some will argue that exceptions can be made on the basis of genuine need - but this only serves to single out students who may have already overcome a great deal to get to class. Consider also that many students may not disclose or even understand their needs, have them officially documented or fully be able to articulate or understand why doing things in a certain way works really well for them.

Technology, from textbooks to overhead projector slides to presentation software, enables information transfer. Lecture capture and laptops permit the recording of information in new and interesting ways. Unfortunately they also lead to content-dense curricula that overwhelm students, obscuring the key concepts and themes, and strategic learning. This actively inhibits students in developing chemical thinking. To learn to think like a chemist — to understand the nature of molecules, their preparation, properties and characterization - is the goal of chemistry education. We need to get better at using technology to facilitate better learning, rather than running from what we do not understand. The rate of innovation across the sector is so fast that I do not believe we will ever fully understand how different technologies impact learning. So there is no point in waiting for that epiphany before trying something new. With a little imagination and the ability to trust students, technology can support all students and those who teach them.

Katherine Haxton is a Senior Lecturer in the School of Chemical and Physical Sciences, Lennard-Jones Building, Keele University, Keele, UK

e-mail: k.j.haxton@keele.ac.uk

doi: $10.1038 / \mathrm{s} 41570.018 .0123$

Published online 28 Feb 2018

Competing interests

The author declares no competing interests.

RELATED LINKS

Richard Feynman's lectures: http://www.cornell.edu/video/ playlist/richard-feynman-messenger-lectures

Learning styles debacle: https://www.theguardian.com/ education $/ 2017 / \mathrm{mar} / 12 / \mathrm{no}$-evidence-to-back-idea-oflearning-styles 